\title{
Which Patients with Obesity Are at Risk for Renal Disease?
}

\author{
Justo Sandino ${ }^{a}$ Leonella Luzardo $^{b}$ Enrique Morales ${ }^{a, c, d}$ Manuel Praga ${ }^{a, c, d}$ \\ aDepartment of Nephrology, University Hospital "12 de Octubre", Madrid, Spain; ${ }^{\text {b} N e p h r o l o g y ~ C e n t e r, ~ H o s p i t a l ~}$

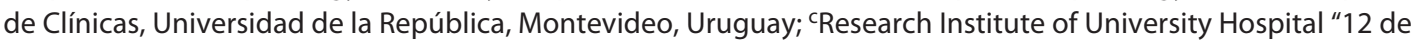 \\ Octubre" (imas12), Madrid, Spain; ${ }^{\text {d} D e p a r t m e n t ~ o f ~ M e d i c i n e, ~ C o m p l u t e n s e ~ U n i v e r s i t y ~ o f ~ M a d r i d, ~ M a d r i d, ~ S p a i n ~}$
}

\section{Keywords}

Obesity $\cdot$ Renal disease $\cdot$ Hyperfiltration $\cdot$ Risk factor

\begin{abstract}
Obesity-related glomerulopathy (ORG) is an increasingly recognized cause of end-stage kidney disease. The most common clinical presentation is a slowly increasing nonnephrotic proteinuria that is followed by a progressive decline of kidney function. Key histological findings are glomerulomegaly and lesions of focal and segmental glomerulosclerosis. A central pathogenic mechanism is the increased sodium reabsorption by proximal tubules that typically accompanies obesity. This causes a decrease in the offer of sodium to the macula densa in the distal nephron, which results in a vasodilation of afferent glomerular arterioles and glomerular hyperfiltration. From a clinical point of view, it is essential to differentiate focal segmental glomerulosclerosis secondary to obesity from primary glomerular processes, which requires a careful differential diagnosis. Diet-induced weight loss, bariatric surgery, and renin-angiotensin blockers are the fundamental therapeutic measures in ORG. The recently developed sodium-glucose cotransporter 2 inhibitors and glucagon-like peptide 1 agonist represent a significant advance in renal protection and will probably improve clinical kidney outcomes in ORG.

(c) 2021 S. Karger AG, Basel
\end{abstract}

\section{Introduction}

The prevalence of obesity is increasing worldwide, and the incidence of obesity-related glomerulopathy (ORG) is becoming one of the leading causes of end-stage kidney disease (ESKD). In this review, we update the epidemiological data of obesity and ORG, summarize the major histological findings, and discuss the pathophysiological mechanisms that explain the mechanistic links between obesity and renal damage. Finally, we propose the different therapeutic options for renal damage associated with obesity.

\section{Epidemiology}

Over the past few decades, overweight and obesity have reached epidemic proportions worldwide, with an increase from 29.8 to $36.9 \%$ in adult men and from 29.8 to $38 \%$ in adult women $[1,2]$. The increase in obesity is particularly worrying in developing countries and in poorer socioeconomic sectors, since its impact on global health and mortality is exacerbated by concomitant fac-

Contribution from the CME course of the DIABESITY Working Group of the ERA-EDTA, Alcorcón (Madrid), Spain, November 15-16, 2019.

$\begin{aligned} & \text { karger@karger.com } \\ & \text { www.karger.com/nef }\end{aligned}$
Karger ${ }^{\prime /}$

Enrique Morales

Department of Nephrology, University Hospital "12 de Octubre"

Avda. Córdoba km 5.400

ES -28041 Madrid (Spain)

emoralesr@senefro.org 
tors such as an inadequate nutrition during pregnancy (leading to a reduced number of nephrons and endocrine pancreas cells at birth), a sedentary lifestyle, and a high prevalence of hypertension and type 2 diabetes. The final result is a perfect environment for the occurrence of chronic kidney disease (CKD) [3].

It remains unclear why only some subjects with obesity develop CKD. Several studies have shown that there are different phenotypes of obesity, metabolically healthy obese and metabolically unhealthy obese. The profile of fat distribution is a key element in this distinction; metabolically healthy obese have more subcutaneous, less visceral fat mass and lower ectopic fat deposition in the liver, kidney, and skeletal muscle than metabolically unhealthy individuals. The components of the metabolic syndrome (hypertension, hypertriglyceridemia, low HDL-cholesterol, and high fasting serum glucose levels) closely correlate with the typical fat distribution of metabolically unhealthy obese, and recent studies have suggested that the occurrence of CKD is associated with metabolic syndrome and visceral fat deposition [3].

This higher risk of CKD and ESKD among patients with obesity does not only affect the adult population. Obese children with obesity have been reported to have larger kidneys, an increased renal flow than nonobese children, and a greater risk to develop ESKD later in life $[4,5]$.

It has been estimated that in $15-30 \%$ of patients with $\mathrm{CKD}$, overweight and obesity play a key pathogenic role [6]. A recent systematic review and meta-analysis of longitudinal cohorts suggested that obesity is an independent predictor for new-onset CKD [7]. Obesity can also negatively influence the prognosis of many kidney diseases with different pathogenic mechanisms. For example, in some entities such as IgA nephropathy, an elevated BMI is associated with hypertension, increased proteinuria, and a greater likelihood of ESKD [8], and recent findings in patients with ADPKD (autosomal dominant polycystic kidney disease) demonstrated that overweight and particularly obesity are strongly associated with rate of progression of early-stage ADPKD, as measured by annual percent change in TKV and eGFR slope [9].

\section{Histology Findings in ORG}

Histology findings in ORG encompass a wide range of heterogeneous lesions. Noteworthy, renal histology in patients with metabolic syndrome and moderate obesity is almost unknown, and the classic histologic lesions are commonly found in patients with severe obesity; this precludes us from precisely describing the histological findings in patients with mild or moderate obesity. Mesangial matrix increase, mesangial cell proliferation, podocyte hypertrophy, and glomerulomegaly are among the most common renal lesions observed [10]. Of these findings, glomerulomegaly is suggested to be a marker of increased risk for progressive renal disease in this group of patients [11]. Alterations often referred as "diabetoid" changes (focal mesangial sclerosis and focal thickening of glomerular and tubular basement membranes) are also frequently seen in patients with obesity without diabetes. In more advanced stages of ORG, lesions of focal segmental glomerulosclerosis (FSGS) can be found, typically affecting hypertrophied glomeruli. They are often perihilar (contiguous with the glomerular vascular pole) but may involve any portion of the glomerulus. Among the 5 histologic subtypes of FSGS (not otherwise specified, perihilar, cellular, tip, and collapsing), ORG exhibits a predominance of the perihilar variant. This predilection for perihilar sclerosis might reflect the greater ultrafiltration pressure (the difference between glomerular capillary hydrostatic and oncotic pressure) at the afferent pole of the glomerular capillary bed. Tubular atrophy and/or interstitial fibrosis and arteriosclerosis are common features, but the tubulointerstitial scarring and inflammation are generally less severe than in primary FSGS. Immunofluorescence reveals nonspecific trapping of IgM and complement C3 in lesions of sclerosis and hyalinosis. By electron microscopy, protein and lipid resorption droplets can be identified focally within podocytes [12].

Other typical features are the presence of an irregular, mild effacement of foot processes, in contrast with the diffuse effacement seen in other glomerular entities causing nephrotic syndrome (in ORG biopsy samples, effacement can be as low as $25 \%$ despite nephrotic-range proteinuria) and the presence of lipid deposits in mesangial and tubular cells [13]. It is noteworthy that patients with ORG may have other coexisting lesions not related to obesity: ORG alone was found in only 29 of 239 (12\%) of renal biopsies from morbidly patients with obesity in a cohort from Columbia University, highlighting the importance of renal biopsy to identify other entities influencing patient management and prognosis. From this series, 44 of 239 (18.4\%) biopsied patients had other renal disease alongside ORG, and the largest group (166 of 239, or 69.5\%) had non-ORG disease alone [14]. 


\section{Pathogenesis of ORG}

A reduction in functional renal mass, regardless of its origin, causes adaptive functional and structural changes in the remaining nephrons. The resulting hyperfiltration is due to vasodilation of the afferent arteriole, which increases the intraglomerular hydrostatic pressure [15]. Both obesity and diabetes are conditions characterized by a hemodynamic hyperfiltration profile, but without reduction in renal mass. Increasing evidence supports that glomerular hyperfiltration plays a central role in the pathogenesis of ORG. Under physiological conditions, most of the sodium filtered into the nephron is reabsorbed in the proximal convoluted tubule. Obesity and diabetes mellitus are associated with an increase in proximal renal sodium reabsorption. Consequently, the macula densa senses less amounts of sodium and initiates a signal pathway that results in preferential vasodilatation of afferent arterioles, with increased glomerular hydrostatic pressure and glomerular filtration rate (GFR) [16]. As hydrostatic pressure in glomerular capillaries increases, the glomerular basement membrane (GBM) enlarges due to elastic expansion and recruitment of membrane wrinkled near the mesangium [17-19]. The resulting podocyte hypertrophy is an adaptive response to the increased GBM. The continuous mechanical challenge to podocyte's foot process finally separates them from the GBM with podocyturia and appearance of segmental scars (FSGS) over the previously enlarged glomerulus.

Of note, the origin of the obesity-related hyperfiltration is the increased proximal tubular sodium (and glucose) reabsorption via sodium-glucose cotransporter 1 and sodium-glucose cotransporter 2 (SGLT1 and SGLT2). The inhibition of the SGLT2 in the proximal segments of the nephrons is in theory an ideal intervention to inhibit the tubulo-glomerular feedback and mitigate glomerular hyperfiltration in obese subjects. The postulated mechanism is that by reducing tubular sodium reabsorption, SGLT2 inhibitors may increase sodium sensed by the macula densa, restore afferent arteriole resistance, and consequently limit glomerular hyperfiltration. Additionally, due to its natriuretic effects, SGLT2 inhibition drugs might diminish volume expansion and contribute to restore intraglomerular pressure in patients with obesity and CKD [20]. SGLT2 inhibitors can lower intraglomerular filtration pressures, which has been attributed to their influence on the proximal reabsorption of sodium [21].

An important issue is the frequent presence of $>1$ clinical condition associated with hyperfiltration on the same patient, for example, a patient with obesity and diabetes mellitus. Hyperfiltration mechanisms will also be enhanced in patients with ORG exposed to a renal mass reduction from any cause. Several studies have demonstrated that the risk of ESKD in patients with extreme renal mass reduction was higher on overweight or patients with obesity as compared with nonobese patients [22, 23].

The examination of long-term renal function in kidney living donors is another clinical model that allows the evaluation of different risk factors for a decline in GFR in patients with a reduced renal mass. Nearly, 40 nonobese and 94 obese living donors per 10,000 developed ESKD after 20 years of follow-up. Kidney donor obesity was a risk factor for the development of ESKD. The absolute risk for postdonation ESKD was low, but compared to nonobese donors, subjects with obesity had a 1.9-fold increased risk for postdonation ESKD [24].

Besides acquired reduction in renal mass, a congenitally reduced nephron endowment may be due to the increasing prevalence of low birth weight, attributed to small for gestational age or preterm birth. The prevalence of low birth weight is high in developing countries and is increasing in most developed countries. Epidemiological studies have shown that the prevalence of proteinuria and/or CKD gradually increases with decreasing birth weights [25]. An analysis of autopsies showed a linear increase in the number of glomeruli up to a birth weight of $3,000 \mathrm{~g}$ [26]. In preterm infants, nephrogenesis continues after birth, but nevertheless they have fewer functional nephrons [25]. The combination of reduced nephron endowment and obesity later in life is an increasing problem with serious medical consequences, particularly in underdeveloped countries.

\section{Which Patients with Obesity Are at Risk for Renal Disease?}

Not all patients with obesity develop CKD, and therefore it is extremely important to identify those who are at a higher risk. How obesity favors progression or how it induces CKD is not fully understood. Obesity defined as BMI $>30 \mathrm{~kg} / \mathrm{m}^{2}$ correlates well with an excess of adipose tissue and is useful in large population studies, but it does not discriminate visceral from subcutaneous fat, or muscle from adipose tissue. Interestingly, some people who are overweight or obese are "metabolically healthy" and protected from cardiometabolic disorders [27]. The metabolically healthy obesity phenotype refers to patients with obesity but with a metabolically healthy state, defined as the absence of any of the metabolic factors (impaired fasting glucose or diabetes, hypertension, hypertriglyceridemia, or low HDL-cholesterol concentration). They have a low bur- 
den of adiposity-related metabolic abnormalities compared with at-risk obese people and fewer metabolic complications of obesity. They are characterized by high insulin sensitivity, low prevalence of hypertension, and a favorable fasting glucose, lipid, and inflammation profile, and their cardiovascular risk appears to be lower than expected for their BMI. On the other hand, metabolically abnormal obesity phenotype is associated with a significantly higher risk of incident proteinuria and/or CKD [28].

The ectopic lipid accumulation in the kidney is called "fatty kidney" or "renal steatosis" and is thought to contribute to the pathophysiology of ORG. Data from in vitro and animal models have shown how lipid accumulation in different types of renal cells could probably affect their structure and function. Lipid deposition in kidney tissue promotes phenotypic changes in podocytes such as insulin-resistant podocytes and podocyte apoptosis. The remaining cells suffer a maladaptive response to glomerulomegaly. As a result of the denudated glomerular tuft, albumin is filtered through the weakened capillary loop, coated with fatty acids, and is reabsorbed by proximal tubule cells leading to hypertrophy, insulin resistance, and increased renal gluconeogenesis [29]. Particularly, renal sinus fat obstructs the blood and lymph outflow of the kidney increasing hydrostatic pressure. The increase of renal volume as well as renal sinus fat volume in prediabetic subjects is associated with visceral adipose tissue and with cardiovascular risk factors [30]. The observation that microalbuminuria and macroalbuminuria do not always precede the loss of renal function in type 2 diabetes has led to the hypothesis that additional factors may play a role in the progression of renal disease before the onset of proteinuria. Other key clinical observations that support this supposition are that reduced GFR without proteinuria is observed in about half of patients with diabetes and renal disease, and renin-angiotensin-aldosterone system inhibitors are less effective in slowing progression of renal disease in type 2 diabetes than in type 1 diabetes patients. Taken together, these observations suggest the presence of nonproteinuric pathways in loss of renal filtration in some patients with type 2 diabetes. It is important to recognize the wide heterogeneity of this disorder because it deserves a personalized therapeutic approach [31].

\section{Clinical Features}

The association between obesity and severe proteinuria has been known for $>40$ years [32]. The magnitude of proteinuria varies widely, ranging from $<1 \mathrm{~g} /$ day to nephrotic range, or even massive proteinuria. The hallmark of ORG is the detection of proteinuria in a patient with obesity with an anodyne urinary sediment $[13,33]$. Characteristically, even in those with nephrotic-range proteinuria ( $>3.5 \mathrm{~g} /$ day), ORG patients do not develop a full nephrotic syndrome with hypoalbuminemia, edema, and hyperlipidemia. Few patients can have massive proteinuria ( $>20 \mathrm{~g} /$ day), but the presence of full nephrotic syndrome is exceptional. From a diagnostic point of view, it is of utmost importance that the clinician correctly differentiates a nephrotic-range proteinuria from a nephrotic syndrome. For example, if a patient with obesity develops sudden onset edema and laboratory studies confirm a plasmatic albumin level below the normal range, a primary glomerular disease that presents as nephrotic syndrome (particularly minimal change disease, primary FSGS, or membranous nephropathy) must be ruled out. On the other hand, failure to differentiate between primary and secondary FSGS may result in many patients undergoing unnecessary and harmful, immunosuppressive treatment. The importance of distinguishing nephrotic-range proteinuria from nephrotic syndrome is especially relevant in patients with FSGS lesion in a renal biopsy [34]. The consequence of this particularly oligosymptomatic clinical presentation is that patients with obesity can develop an unperceived proteinuria for years and only be detected at an advanced renal disease stage. The reason why ORG patients do not develop nephrotic syndrome in spite of the presence of massive proteinuria is unknown. The slow progression of proteinuria (along years) might allow an increase in hepatic synthesis of albumin, acting as a compensating mechanism that limits its impact [35]. Other possible explanation is that tubular degradation and reabsorption of filtered proteins can be different in nephropathies caused by hyperfiltration, as compared to glomerular diseases that cause full nephrotic syndrome. Other hyperfiltration-induced renal diseases, such as reflux nephropathy or FSGS-associated renal mass reduction, also present as massive proteinuria without nephrotic syndrome. Recent studies in murine models have supported the hypothesis that proteinuria stimulates $\mathrm{Na}$ reabsorption in the aldosterone-sensitive distal nephron. This Na reabsorption is sensitive to blockers of the epithelial sodium channel, suggesting the epithelial sodium channel plays a role in proteinuria-associated $\mathrm{Na}$ retention, partially explaining the lack of edema in these patients [36]. The lack of edema ends up very frequently with a delay in the detection of proteinuria, and no specific studies have been conducted to investigate the influence of the level of proteinuria on the progression of renal 
damage among patients with ORG. However, the available data suggest that the relationship between proteinuria level and progression of renal damage could also been applied to this entity: reported patients with obesityrelated FSG and a progression into end-stage renal failure usually show very high levels of proteinuria, whereas those showing only mild proteinuria or microalbuminuria usually exhibit a stable renal function [37]. Misinterpretation between primary and secondary FSGS is particularly relevant in African-Americans, whom some authors postulate have a different disease from primary FSGS seen in Caucasians [35]. The majority of hypertensive African-Americans patients do not show FSGS, but rather focal global glomerulosclerosis, arteriolosclerosis, and/or arteriosclerosis are almost universal and EM often shows only segmental foot process effacement. Clinical presentation is usually without nephrotic syndrome and subnephrotic-range proteinuria. The condition is associated with variants in the APOL1 gene that correlate with the accelerated rate of decline in kidney function in these patients [35, 38].

\section{Treatment}

Both weight loss and RAS blockade are effective in the treatment of ORG. Weight loss is usually accompanied by a reduction of proteinuria, not only in ORG, but also in patients with other proteinuric nephropathies. Interestingly, the greater the weight loss, the greater the reduction in proteinuria. A sedentary lifestyle combined with highcalorie diets has resulted in an alarming prevalence of obesity. Attempts to decrease body weight have been largely concentrated on lifestyle interventions and lowcalorie diets with modest results. The Look AHEAD randomized clinical trial analyzed the effect of a long-term lifestyle intervention on nephropathy, in patients with type 2 diabetes. The intensive lifestyle intervention group achieved sustained reduction in body weight, $\mathrm{HbAlc}$, and blood pressure. The incidence rate of very high-risk CKD (according to the 2012 KDIGO classification) over a follow-up of $>9$ years was $31 \%$ lower compared to the diabetes support and education group. This outcome suggests that weight loss should be considered in addition to other therapies to delay the progression of CKD in patients with obesity with type 2 diabetes [39].

A novel approach regarding low-calorie diets is the finding that intermittent fasting (exposure to fasting periods) increases insulin sensitivity, improves lipid metabolism, and reduces abdominal fat and inflammation, among other metabolic benefits [40]. Periods of energy restriction, long enough to deplete liver glycogen stores, activate a metabolic switch toward use of fatty acids and ketones. Cell adaptation consists of a reduction in insulin signaling and overall protein synthesis. During the recovery phase, glucose plasma levels increase, ketone levels drop, and cells increase protein synthesis [40].

Bariatric surgery is much more effective in weight loss than low-calorie diets and is now an accepted treatment option for severe obesity. This therapeutic approach has beneficial effects on long-term obesity-related comorbidities, including renal disease. A recent study proposes that the impact of bariatric surgery on type 2 diabetic patients may depend on age and found that adolescents showed faster resolution of elevated urine albumin-to-creatinine ratio following gastric bypass compared with adults [41]. One aspect to carefully contemplate is the fact that the rate of perisurgical complications is usually higher in patients with the double condition of morbid obesity and renal disease. Patients with serum creatinine $>2 \mathrm{mg} / \mathrm{dL}$ before the surgery have a higher risk of reintervention, readmission, and severe kidney injury. Nevertheless, the absolute risk is still low, and mortality risk does not increase [42]. On a prospective cohort study, bariatric surgery resulted in lower CKD risk after a 7-year follow-up period. Reduction in risk was most pronounced in persons with high baseline risk; nevertheless, improvements were also observed in patients with moderate risk at enrollment. Authors emphasize consideration of CKD risk in evaluation for bariatric surgery and study bariatric surgery as a treatment for high-risk patients with obesity and CKD [43]. There are 2 prospective trials ongoing (NCT Nos. 02644928 and 01410994) specifically designed to analyze the relationship between changes in weight loss (induced by bariatric surgery) and proteinuria.

Bariatric surgery induces a decrease in adipokines like leptin and visfatin related with obesity-induced metabolic disorders. Importantly, circulating levels of adiponectin, an adipokine with protective effects on kidney function, increased 12 months after bariatric surgery. Adiponectin is downregulated in obesity, and numerous experimental studies have reported anti-inflammatory, antifibrotic, and antioxidant properties of this adipokine, together with a reduction in albuminuria. These changes in metabolic processes, pro-inflammatory mediators, and adipocyte cytokines can contribute to improved renal injury regardless of weight loss [44].

There are several factors that develop cardio and nephroprotection after bariatric surgery, including reduced sympathetic drive, remission of glomerular hyper- 
tension, increased natriuresis, changes in the gut microbiota, and reduction of systemic and renal inflammation, increasing the functionality of high-density lipoproteins and reducing adverse cardiac remodeling [45].

Among the pathogenic mechanisms and hemodynamic alterations in obesity, the increase in sodium reabsorption in the proximal tubule plays a fundamental role. Docherty et al. [46], in a nice clinical study, showed us how patients with obesity developed an increased renal fractional excretion of sodium after bariatric surgery. This finding highlights the gut-kidney axis and its possible clinical implications [46].

Finally, the role of bariatric surgery in achieving remission of diabetes mellitus is unquestionable; however, a percentage of these patients do not achieve this long-term remission. For this reason, the management of persistent or recurrent type 2 diabetes after metabolic surgery represents a clinical challenge such as the use of glucose-lowering medication. Recently, the results of the GRAVITAS randomized clinical trial, the use of liraglutide, a glucagon-like peptide 1 analog in patients with persistent or recurrent type 2 diabetes after bariatric surgery, have been published. The authors of this study have shown that the use of the GLP-1 receptor agonist can be safe and effective in improving glycemia and bodyweight in this patient population [47].

It is well recognized that albuminuric nephropathies progression is slowed by the use of angiotensin-converting enzyme inhibitors or angiotensin receptor blockers [48], and several observational studies have shown a significant antiproteinuric effect. Since this observation 20 years ago, there have been many attempts to describe drugs that further slow diabetic kidney disease, but all have failed, even with some of them increasing morbidity [49].

Recently, an event of great impact that has motivated a change of the treatment paradigm of diabetes was the introduction of sodium-glucose cotransporter 2 inhibitor (SGLT2i) and glucagon-like peptide 1 receptor agonist (GLP-1 RA). These promising treatments are capable to prevent cardiovascular events and kidney failure on patients with already high cardiovascular and renal risk. In particular, SGLT2i showed outstanding effects to slow the decline of glomerular function rate and reduce the progression to end-stage renal disease in patients with diabetes that were already taking RAS inhibitors. The renoprotective effect of SGLT2i is of great interest in clinical practice, in patients who combine a high cardiovascular risk and kidney disease. SGLT2 inhibitors reduced the risk of dialysis, transplantation, or death due to kidney disease, and remarkably renoprotection was achieved across different levels of kidney function, even for the subgroup with baseline eGFR between 30 and $45 \mathrm{~mL} / \mathrm{min}$ [50, 51].

A systematic review and meta-analysis of randomized, controlled trials of SGLT2i that reported effects on kidney outcomes analyzed empagliflozin (EMPA-REG OUTCOME), canagliflozin (CANVAS Program and CREDENCE), and dapagliflozin (DECLARE-TIMI 58) trials. SGLT2 inhibitors reduced the risk of dialysis, transplantation, or death due to kidney disease (RR 0.67, 95\% CI $0.52-0.86, p=0.0019)$. ESKD was also reduced $(0.65$, $0.53-0.81, p<0.0001)$. Renoprotection was present irrespective of baseline albuminuria and use of RAS blockade [50].

As SGLT2i, the glucagon-like peptide 1 analog appears to have glucose-independent effects on diabetic nephropathy progression. The GLP-1 receptor agonists improve metabolic control by stimulating insulin and suppressing glucagon secretion, but also have extra pancreatic actions including renal effects. The Liraglutide Effect and Action in Diabetes: Evaluation of Cardiovascular Outcome Results (LEADER) trial studied the long-term effects of liraglutide on renal outcomes in patients with type 2 diabetes. The composite renal outcome was defined as a composite of new-onset persistent macroalbuminuria, persistent doubling of the serum creatinine level, end-stage renal disease, or death due to renal disease. Among patients with type 2 diabetes who were at high cardiovascular risk, added to usual care, liraglutide resulted in significantly lower rates of renal outcomes than placebo. This was driven mostly by a lower incidence of new-onset persistent macroalbuminuria in the liraglutide group than in the placebo group. There were no significantly lower risks of end-stage renal disease with liraglutide than with placebo during up to 5 years of follow-up. The eGFR declined continuously on both groups of patients, but the decline was slightly slower in the liraglutide group [52]. Given that diabetic nephropathy and ORG share multiple features of their pathophysiology, these results achieved in patients with type 2 diabetes could be extrapolated to patients with ORG.

The renal tubular effects of GLP-1 receptor agonists (GLP-1RA) include an increase in absolute and fractional excretions of sodium, chloride, and potassium and increased urinary $\mathrm{pH}$. Experimental data suggest that this effect could be mediated at least in part by an inhibitory effect on the sodium-hydrogen exchanger isoform 3 (NHE3) in the proximal tubule. Patients treated during 8 weeks with lixisenatide, a short-acting GLP-1 receptor antagonist, had significantly more phosphorylated 
NHE3 in urinary extracellular vesicles than those on insulin glulisine treatment, which suggested decreased NHE3 activity in the proximal tubule [53, 54]. A placebo controlled clinical trial designed to investigate the acute kidney effects of exenatide, another GLP-1RA, demonstrated that the acute intravenous administration did not affect renal hemodynamics in patients with obesity and type 2 diabetes. Authors found that compared to placebo, exenatide increased absolute and fractional sodium excretion [55].

A relevant role for nondiabetic indications of SGLT2 inhibitors is emerging due to increasing evidence of an improvement in cardiovascular and renal outcomes in patients without diabetes. Benefits beyond glucose lowering are being supported by milestone trials, such as DAPA-HF. In patients with heart failure and an ejection fraction of $40 \%$ or less, dapagliflozin reduced hospitalization due to worsening heart failure or cardiovascular death compared to placebo, regardless of the presence or absence of diabetes [56]. The effect of dapagliflozin in patients with CKD, with or without type 2 diabetes, randomly assigned to receive dapagliflozin or placebo was recently communicated. The trial was stopped earlier because of efficacy. Over a median of 2.4 years, a primary outcome event (a composite of a sustained decline in the estimated GFR of at least $50 \%$, ESKD, or death from renal or cardiovascular causes) occurred in $9.2 \%$ in the dapagliflozin group and $14.5 \%$ in the placebo group [57].
More long-term clinical trials designed to investigate the effects of SGLT2 inhibitors in patients without diabetes are needed, but it is reasonable to speculate that more patients can benefit from these drugs. A careful patient selection by a risk assessment algorithm will mitigate adverse effects and facilitate the acceptance of SGLT2i and GLP-1 RA for heart and kidney protection. When a patient has concomitant heart failure and CKD, SGLT2i is preferred. However, for patients with high metabolic risks or when eGFR is $<30 \mathrm{~mL} / \mathrm{min}$ per $1.73 \mathrm{~m}^{2}$, GLP-1 $\mathrm{RA}$ is a better option [58].

\section{Conflict of Interest Statement}

The authors have no conflicts of interest to declare.

\section{Funding Sources}

This work has been made possible through ISN-funded fellowship to L.L. E.M. holds a research grant (2015/0117) "Efectos de la pérdida de peso sobre la función renal en pacientes obesos con enfermedad renal" from Fundación SENEFRO.

\section{Author Contributions}

All the authors contributed equally to this study, revised the article, and approved the final version of the manuscript.

\section{References}

1 Wang Y, Chen X, Song Y, Caballero B, Cheskin LJ. Association between obesity and kidney disease: a systematic review and meta-analysis. Kidney Int. 2008;73(1):1933.

2 Swinburn BA, Kraak VI, Allender S, Atkins VJ, Baker PI, Bogard JR, et al. The global syndemic of obesity, undernutrition, and climate change: the Lancet Commission report. Lancet. 2019;393(10173):791-846.

3 Stefan N, Häring HU, Hu FB, Schulze MB. Metabolically healthy obesity: epidemiology, mechanisms, and clinical implications. Lancet Diabetes Endocrinol. 2013; 1(2):152-62.

4 Ding W, Cheung WW, Mak RH. Impact of obesity on kidney function and blood pressure in children. World J Nephrol. 2015; 4(2):223-9.

5 Correia-Costa L, Azevedo A, Caldas Afonso A. Childhood obesity and impact on the kidney. Nephron. 2019;143(1):8-11.
6 Rhee CM, Ahmadi SF, Kalantar-Zadeh K. The dual roles of obesity in chronic kidney disease: a review of the current literature. Curr Opin Nephrol Hypertens. 2016;25(3): 208-16.

7 Garofalo C, Borrelli S, Minutolo R, Chiodini P, De Nicola L, Conte G. A systematic review and meta-analysis suggests obesity predicts onset of chronic kidney disease in the general population. Kidney Int. 2017; 91(5):1224-35.

8 Berthoux F, Mariat C, Maillard N. Overweight/obesity revisited as a predictive risk factor in primary $\operatorname{IgA}$ nephropathy. Nephrol Dial Transplant. 2013;28(Suppl 4):iv160-6.

9 Nowak KL, You Z, Gitomer B, Brosnahan G, Torres VE, Chapman AB, et al. Overweight and obesity are predictors of progression in early autosomal dominant polycystic kidney disease. J Am Soc Nephrol. 2018 Feb;29(2):571-8.
10 Hughson M, Farris AB, Douglas-Denton R, Hoy WE, Bertram JF. Glomerular number and size in autopsy kidneys: the relationship to birth weight. Kidney Int. 2003; 63(6):2113-22.

11 D’Agati VD, Chagnac A, de Vries AP, Levi $M$, Porrini E, Herman-Edelstein $M$, et al. Obesity-related glomerulopathy: clinical and pathologic characteristics and pathogenesis. Nat Rev Nephrol. 2016;12(8):45371.

12 D’Agati VD, Kaskel FJ, Falk RJ. Focal segmental glomerulosclerosis. N Engl J Med. 2011;365:2398-411.

13 Kambham N, Markowitz GS, Valeri AM, Lin J, D'Agati VD. Obesity-related glomerulopathy: an emerging epidemic. Kidney Int. 2001;59(4): 1498-509.

14 Choung H-YG, Bomback AS, Stokes MB, Santoriello D, Campenot ES, Batal I, et al. The spectrum of kidney biopsy findings in patients with morbid obesity. Kidney Int. 2019 Mar;95(3):647-54. 
15 Anderson S, Meyer TW, Rennke HG, Brenner BM. Control of glomerular hypertension limits glomerular injury in rats with reduced renal mass. J Clin Invest. 1985 Aug;76(2):612-9.

16 Chagnac A, Herman M, Zingerman B, Erman A, Rozen-Zvi B, Hirsh J, et al. Obesity induced glomerular hyperfiltration: its involvement in the pathogenesis of tubular sodium reabsorption. Nephrol Dial Transplant. 2008 Dec;23(12):3946-52.

17 Denic A, Mathew J, Lerman LO, Lieske JC, Larson JJ, Alexander MP, et al. Singlenephron glomerular filtration rate in healthy adults. N Engl J Med. 2017 Jun; 376(24):2349-57.

18 Chagnac A, ZIngerman B, Rozen-Zvi B, Herman-Edelstein M. Consequences of glomerular hyperfiltration: the role of physical forces in the pathogenesis of chronic kidney disease in diabetes and obesity. Nephron. 2019;143(1):38-42.

$19 \mathrm{Kriz}$ W, Lemley KV. A potential role for mechanical forces in the detachment of podocytes and the progression of CKD. J Am Soc Nephrol. 2015 Feb;26(2):258-69.

20 Sarafidis P, Ferro CJ, Morales E, Ortiz A, Malyszko J, Hojs R, et al. SGLT-2 inhibitors and GLP-1 receptor agonists for nephroprotection and cardioprotection in patients with diabetes mellitus and chronic kidney disease. A consensus statement by the EURECA-m and the DIABESITY working groups of the ERA-EDTA. Nephrol Dial Transplant. 2019 Feb;34(2):208-30.

21 Layton AT, Vallon V, Edwards A. Modeling oxygen consumption in the proximal tubule: effects of NHE and SGLT2 inhibition. Am J Physiol Renal Physiol. 2015 Jun; 308(12):F1343-57.

22 Praga M, Hernández E, Herrero JC, Morales E, Revilla Y, Díaz-González R, et al. Influence of obesity on the appearance of proteinuria and renal insufficiency after unilateral nephrectomy. Kidney Int. 2000 Nov;58(5):2111-8.

23 González E, Gutiérrez E, Morales E, Hernández E, Andres A, Bello I, et al. Factors influencing the progression of renal damage in patients with unilateral renal agenesis and remnant kidney. Kidney Int. 2005 Jul;68(1):263-70.

24 Locke JE, Reed RD, Massie A, MacLennan PA, Sawinski D, Kumar V, et al. Obesity increases the risk of end-stage renal disease among living kidney donors. Kidney Int. 2017 Mar;91(3):699-703.

25 Kanda T, Murai-Takeda A, Kawabe H, Itoh H. Low birth weight trends: possible impacts on the prevalences of hypertension and chronic kidney disease [published online ahead of print, 2020 May 11]. Hypertens Res. 2020;43(9):859-68.
26 Manalich R, Reyes L, Herrera M, Melendi C, Fundora I. Relationship between weight at birth and the number and size of renal glomeruli in humans: a histomorphometric study. Kidney Int. 2000 Aug;58(2):770-3.

27 Hall JE, do Carmo JM, da Silva AA, Wang Z, Hall ME. Obesity, kidney dysfunction and hypertension: mechanistic links. Nat Rev Nephrol. 2019 Jun;15(6):367-85.

28 Hashimoto Y, Tanaka M, Okada H, Senmaru T, Hamaguchi $M$, Asano $M$, et al. Metabolically healthy obesity and risk of incident CKD. Clin J Am Soc Nephrol. 2015 Apr; 10(4):578-83.

29 de Vries AP, Ruggenenti P, Ruan XZ, Praga M, Cruzado JM, Bajema IM, et al. Fatty kidney: emerging role of ectopic lipid in obesity-related renal disease. Lancet Diabetes Endocrinol. 2014 May;2(5):417-26.

30 Notohamiprodjo M, Goepfert M, Will S, Lorbeer R, Schick F, Rathmann W, et al. Renal and renal sinus fat volumes as quantified by magnetic resonance imaging in subjects with prediabetes, diabetes, and normal glucose tolerance. PLoS One. 2020 Feb;15(2):e0216635.

31 Porrini E, Ruggenenti P, Mogensen CE, Barlovic DP, Praga M, Cruzado JM, et al. Non-proteinuric pathways in loss of renal function in patients with type 2 diabetes. Lancet Diabetes Endocrinol. 2015 May; 3(5):382-91.

32 Weisinger JR, Kempson RL, Eldridge FL, Swenson RS. The nephrotic syndrome: a complication of massive obesity. Ann Intern Med. 1974;81(4):440-7.

33 Praga M, Hernández E, Morales E, Campos AP, Valero MA, Martínez MA, et al. Clinical features and long-term outcome of obesity-associated focal segmental glomerulosclerosis. Nephrol Dial Transplant. 2001 Sep;16(9):1790-8.

34 Sethi S, Glassock RJ, Fervenza FC. Focal segmental glomerulosclerosis: towards a better understanding for the practicing nephrologist. Nephrol Dial Transplant. 2015 Mar;30(3):375-84.

35 Praga M, Morales E. The fatty kidney: obesity and renal disease. Nephron. 2017; 136(4):273-6.

36 Bohnert BN, Menacher M, Janessa A, Wörn $\mathrm{M}$, Schork A, Daiminger S, et al. Aprotinin prevents proteolytic epithelial sodium channel $(\mathrm{ENaC})$ activation and volume retention in nephrotic syndrome. Kidney Int. 2018;93(1):159-72.

37 Praga M, Morales E. Weight loss and proteinuria. Contrib Nephrol. 2006;151:2219.

38 Fogo A, Breyer JA, Smith MC, Cleveland WH, Agodoa L, Kirk KA, et al. Accuracy of the diagnosis of hypertensive nephrosclerosis in African Americans: a report from the African American Study of Kidney Disease (AASK) Trial. AASK Pilot Study investigators. Kidney Int. 1997 Jan;51(1): 244-52.
39 Look AHEAD Research Group, Yeh HC, Bantle JP, Cassidy-Begay M, Blackburn G, Bray GA, et al. Intensive weight loss intervention and cancer risk in adults with type 2 diabetes: analysis of the Look AHEAD randomized clinical trial. Obesity. 2020; 28(9):1678-86.

40 de Cabo R, Mattson MP. Effects of intermittent fasting on health, aging, and disease. N Engl J Med. 2019 Dec;381(26): 2541-51.

41 Bjornstad P, Nehus E, Jenkins T, Mitsnefes M, Moxey-Mims M, Dixon JB, et al. Fiveyear kidney outcomes of bariatric surgery differ in severely obese adolescents and adults with and without type 2 diabetes. Kidney Int. 2020 May;97(5):995-1005.

42 Friedman AN, Cohen RV. Bariatric surgery as a renoprotective intervention. Curr Opin Nephrol Hypertens. 2019 Nov;28(6): 537-44.

43 Friedman AN, Wahed AS, Wang J, Courcoulas AP, Dakin G, Hinojosa MW, et al. Effect of bariatric surgery on CKD risk. J Am Soc Nephrol. 2018 Apr;29(4):1289300.

44 Scheurlen KM, Probst P, Kopf S, Nawroth PP, Billeter AT, Müller-Stich BP. Metabolic surgery improves renal injury independent of weight loss: a meta-analysis. Surg Obes Relat Dis. 2019;15(6):1006-20.

45 Martin WP, Docherty NG, Le Roux CW. Impact of bariatric surgery on cardiovascular and renal complications of diabetes: a focus on clinical outcomes and putative mechanisms. Expert Rev Endocrinol Metab. 2018;13(5):251-62.

46 Docherty NG, Fändriks L, le Roux CW, Hallersund P, Werling M. Urinary sodium excretion after gastric bypass surgery. Surg Obes Relat Dis. 2017;13(9):1506-14.

47 Miras AD, Pérez-Pevida B, Aldhwayan M, Kamocka A, McGlone ER, Al-Najim W, et al. Adjunctive liraglutide treatment in patients with persistent or recurrent type 2 diabetes after metabolic surgery (GRAVITAS): a randomised, double-blind, placebo-controlled trial. Lancet Diabetes Endocrinol. 2019;7(7):549-59.

48 Brenner BM, Cooper ME, de Zeeuw D, Keane WF, Mitch WE, Parving HH, et al. Effects of losartan on renal and cardiovascular outcomes in patients with type 2 diabetes and nephropathy. N Engl J Med. 2001; 345(12):861-9.

49 Bakris GL. Major advancements in slowing diabetic kidney disease progression: focus on SGLT2 inhibitors. Am J Kidney Dis. 2019 Nov;74(5):573-5.

50 Neuen BL, Young T, Heerspink HJL, Neal B, Perkovic V, Billot L, et al. SGLT2 inhibitors for the prevention of kidney failure in patients with type 2 diabetes: a systematic review and meta-analysis. Lancet Diabetes Endocrinol. 2017 Nov;7(11):845-54. 
51 Packer M. Role of impaired nutrient and oxygen deprivation signaling and deficient autophagic flux in diabetic CKD development: implications for understanding the effects of sodium-glucose cotransporter 2-inhibitors. J Am Soc Nephrol. 2020 May; 31(5):907-19.

52 Mann JFE, Ørsted DD, Brown-Frandsen K, Marso SP, Poulter NR, Rasmussen S, et al. Liraglutide and renal outcomes in type 2 diabetes. N Engl J Med. 2017;377(9):83948.

53 Gutzwiller JP, Tschopp S, Bock A, Zehnder CE, Huber AR, Kreyenbuehl M, et al. Glucagon-like peptide 1 induces natriuresis in healthy subjects and in insulin-resistant obese men. J Clin Endocrinol Metab. 2004; 89(6):3055-61.
54 Tonneijck L, Muskiet MHA, Blijdorp CJ, Smits MM, Twisk JW, Kramer MHH, et al. Renal tubular effects of prolonged therapy with the GLP-1 receptor agonist lixisenatide in patients with type 2 diabetes mellitus. Am J Physiol Renal Physiol. 2019; 316(2):F231-40.

55 Tonneijck L, Smits MM, Muskiet MHA, Hoekstra T, Kramer MHH, Danser AHJ, et al. Acute renal effects of the GLP-1 receptor agonist exenatide in overweight type 2 diabetes patients: a randomised, double-blind, placebo-controlled trial. Diabetologia. 2016;59(7):1412-21.
56 McMurray JJV, Solomon SD, Inzucchi SE, Køber L, Kosiborod MN, Martinez FA, et al. Dapagliflozin in patients with heart failure and reduced ejection fraction. $\mathrm{N}$ Engl J Med. 2019;381(21):1995-2008.

57 Heerspink HJL, Stefánsson BV, CorreaRotter R, Chertow GM, Greene T, Hou FF, et al. DAPA-CKD trial committees and investigators. Dapagliflozin in patients with chronic kidney disease. N Engl J Med. 2020; 383(15):1436-46.

58 Li J, Albajrami O, Zhuo M, Hawley CE, Paik JM. Decision algorithm for prescribing SGLT2 inhibitors and GLP-1 receptor agonists for diabetic kidney disease. Clin J Am Soc Nephrol. 2020;15(11):1678-88. 